\title{
Pulse radiolysis of liquid water using picosecond electron pulses produced by a table-top terawatt laser system
}

\author{
Ned Saleh, Kirk Flippo, Koshichi Nemoto, and Donald Umstadter \\ Center for Ultrafast Optical Science, University of Michigan, 2200 Bonisteel Boulevard, \\ Ann Arbor, Michigan 48109-2099
}

Robert A. Crowell, ${ }^{\text {a) }}$ Charles D. Jonah, and Alexander D. Trifunac

Chemistry Division, Argonne National Laboratory, Argonne, Illinois 60439

(Received 13 December 1999; accepted for publication 8 February 2000)

\begin{abstract}
A laser based electron generator is shown, for the first time, to produce sufficient charge to conduct time resolved investigations of radiation induced chemical events. Electron pulses generated by focussing terawatt laser pulses into a supersonic helium gas jet are used to ionize liquid water. The decay of the hydrated electrons produced by the ionizing electron pulses is monitored with $0.3 \mu \mathrm{s}$ time resolution. Hydrated electron concentrations as high as $22 \mu \mathrm{M}$ were generated. The results show that terawatt lasers offer both an alternative to linear accelerators and a means to achieve subpicosecond time resolution for pulse radiolysis studies. (c) 2000 American Institute of Physics. [S0034-6748(00)01306-X]
\end{abstract}

\section{INTRODUCTION}

The first observable species following interaction of energetic radiation with condensed matter are radical cations and ejected electrons. The ultrafast thermalization process of these species plays a critical role in determining the final chemical outcome of a radiation induced event. For this reason, the development of subpicosecond pulse radiolysis techniques has been identified as the single most important technological advancement that is needed to enhance the field of radiation chemistry. ${ }^{1}$ Experiments utilizing subpicosecond time resolution will allow the direct observation of the complex chemical and physical processes that are produced by ionizing radiation.

It has been proposed that the most appropriate approach towards the realization of subpicosecond pulse radiolysis is through the development of photocathode-driven linacs (see, for example, Ref. 2). Several photocathode accelerators are under development or at the proposal stage. ${ }^{1,2}$ The challenges and difficulties with photocathode linacs for the measurement of ultrafast processes have to do with (1) pulse stability of the laser used to drive the photocathode and (2) the high degree of time synchronization of the rf field with the laser pulse $(<0.5 \mathrm{ps}){ }^{3}$ Whether one can pursue the study of ultrafast processes induced by ionizing radiation remains a question that will not be answerable for several years until ultrafast pulse radiolysis tools are realized. However, one can partially shorten this developmental time frame by exploring the capabilities of laser-based table-top electron generation. ${ }^{4-6}$ This is a logical stepping stone towards exploration of the same processes that motivate the development of ultrafast pulse radiolysis accelerators.

Recently, significant advances have been made in the field of high power lasers (for a review, see Ref. 7). Of

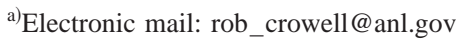

interest here is the ability to amplify subpicosecond pulses up to energies of $0.1 \mathrm{~J}$ or higher. These table-top laser systems are capable of generating peak powers in excess of $10^{12} \mathrm{~W}$ (e.g., $50 \mathrm{fs}, 50 \mathrm{~mJ}$ ). It has been shown that by focusing terawatt $\left(10^{12} \mathrm{~W}\right)$ laser pulses to intensities $\geqslant 10^{18} \mathrm{~W} / \mathrm{cm}^{2}$ in helium gas jets, it is possible to generate electron pulses with a charge of a few $\mathrm{nC}^{4-6}$ While physicists are interested in this technology to study such phenomena as the generation of superrelativistic electrons and nonlinear quantum electrodynamics, chemists could use these techniques to conduct subpicosecond pulse radiolysis experiments.

To exploit the benefits of laser-based electron generators, two main issues must be addressed: (1) the generation of subpicosecond electron pulses with sufficient charge to generate an identifiable quantity of chemical species and (2) the development of sensitive ultrafast detection techniques to observe and characterize the resultant radiolysis transients. It is the purpose of this article to address the first of these issues. We present the results from a pulse radiolysis study of liquid water using electron pulses produced by a table-top terawatt laser system $\left(T^{3}\right)$. The results demonstrate that a $T^{3}$ based electron accelerator is capable of generating sufficient charge to produce a measurable amount of hydrated electron $\left(e_{\mathrm{aq}}^{-}\right)$in liquid water.

\section{EXPERIMENT}

The laser system used to generate the subpicosecond electron pulses is a hybrid Ti:sapphire-Nd:glass laser amplifier system that is capable of producing $5 \mathrm{~J} 400 \mathrm{fs}(12.5 \mathrm{TW})$ pulses at $1053 \mathrm{~nm}$. The basic laser layout is shown in Fig. 1. Briefly, the output of a Ti:sapphire laser (Coherent Mira, $1053 \mathrm{~nm}, 100 \mathrm{fs}, 2 \mathrm{~nJ}$ ) is passed through a standard grating stretcher to increase the pulsewidth to $\sim 1 \mathrm{~ns}$. The pulse energy is then increased to $1 \mathrm{~mJ}$ in a Nd:YAG pumped Ti:sapphire regenerative amplifier. The laser pulses are then passed 


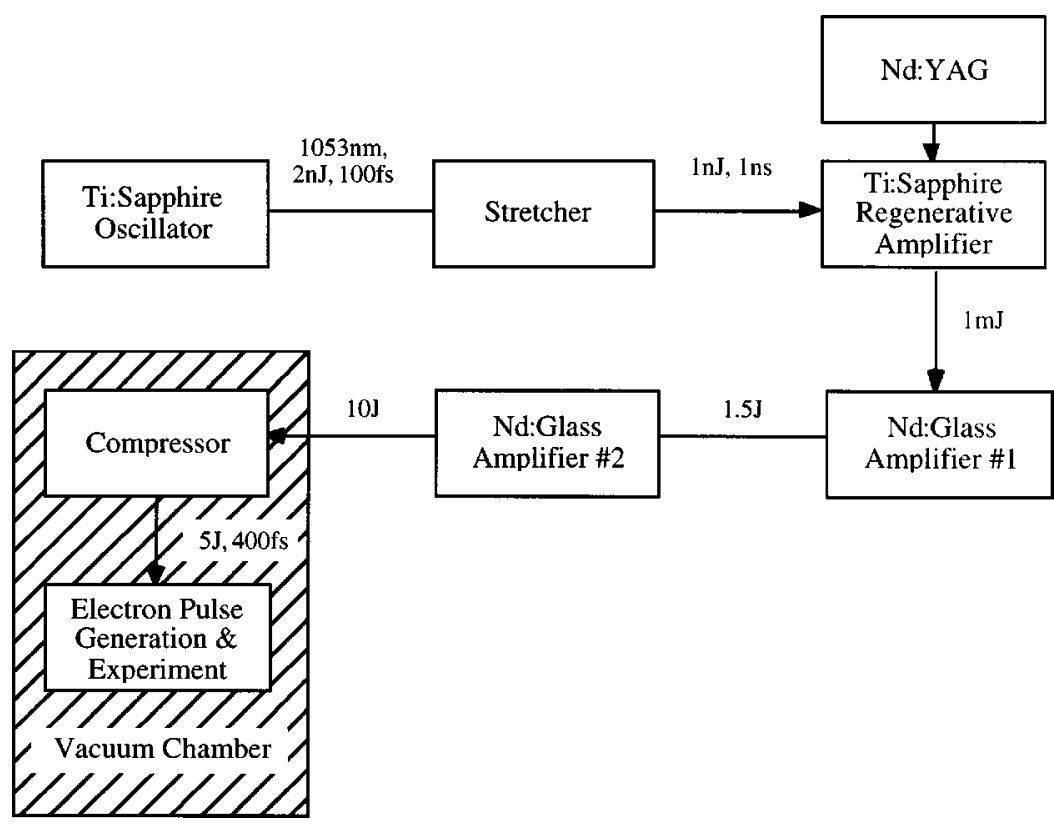

FIG. 1. Block diagram of the laser system. The laser is capable of generating up to $5 \mathrm{~J}$ in a $400 \mathrm{fs}$ pulse centered at $1053 \mathrm{~nm}$. The compressor and all subsequent optics are kept under a vacuum of a few mbar. The repetition rate of the laser is $1 \mathrm{shot} / 10 \mathrm{~min}$.

through two-flash-lamp pumped Nd:glass rod amplifiers, which increase the energy to 1.5 and $10 \mathrm{~J}$, respectively. A series of spatial filters in the amplifier system are used to maintain a Gaussian beam profile and reduce ASE. Pockel cells are used between each amplification stage to prevent damage in the amplifiers due to any back reflections. After amplification, the pulses are compressed to their transform limited value of $400 \mathrm{fs}$ in a standard grating compressor. The compressor and all subsequent optics are under a vacuum of a few mbar. The low repetition rate of the laser, $0.002 \mathrm{~Hz}(1$ shot/10 $\mathrm{min}$ ) requires the implementation of single shot detection schemes. Additional details of the laser system can be found elsewhere. ${ }^{5}$

The pulse energy used to generate the electron pulses was $2 \mathrm{~J}$. The $50 \mathrm{~mm}$ diameter laser beam was focused by a $f / 3$ off-axis parabolic mirror to a spot size of $\sim 9 \mu \mathrm{m}\left(e^{-2}\right.$ intensity) in a supersonic helium gas jet. The maximum intensity was $4 \times 10^{18} \mathrm{~W} / \mathrm{cm}^{2}$. Under these conditions, a collimated beam of $2 \mathrm{MeV}$ electrons is emitted in the laser propagation direction. ${ }^{4-6}$ The spectrum of the electrons is shown in Fig. 2. The divergence of the electron beam was $5.7^{\circ}$.

The temporal (longitudinal) profile of the electron pulses has not been measured directly. The temporal profile of the macrobunch is believed to be approximately equal to that of the plasma wave which has been measured to be $\sim 1.9$ ps. $^{5}$ The separation between microbunches is equal to the plasma wave period which is 18 fs for a plasma density of 3.4 $\times 10^{19} \mathrm{~cm}^{-3}$. The duration of each microbunch is less than $18 \mathrm{fs} .{ }^{5}$ Because of the large dispersion in the electron energy, the temporal profile is expected to increase as the pulse propagates. The combination of dispersion effects and low repetition rate make quantitative characterization of the electron pulse temporal profile difficult.

The experimental setup used to monitor the solvated electrons is shown in Fig. 3. The $e_{\mathrm{aq}}^{-}$are produced by passing the electron pulse through a $1.0 \times 0.5 \mathrm{~cm}$ suprasil cell containing doubly deionized water. A $100 \mu \mathrm{m}$ thick aluminum foil was mounted on the cell window to filter out the 1053 $\mathrm{nm}$ pump photons. The $670 \mathrm{~nm} 3 \mathrm{~mW}(\mathrm{cw})$ output from a diode laser was passed through the sample cell perpendicular to the electron beam and was used to monitor the amount of solvated electrons produced. The diode laser beam overlapped the electron beam $7 \mathrm{~mm}$ downstream from the helium gas jet. At this distance from the gas jet, the electron-beam diameter was $1.2 \mathrm{~mm}$. The diode laser beam was directed into an amplified silicon photodiode whose output was monitored on a digital oscilloscope. This setup allowed for the measurement of transients with transmission changes in the probe beam as small as $\Delta T \sim 0.5 \%$ for a single laser shot. The instrument response for each transient was determined from the rise time of the $e_{\mathrm{aq}}^{-}$absorption signal and was Gaussian with full width at half maximum (FWHM) of 0.3 $\mu \mathrm{s}$. The water was purified in a Barnstead Nanopure cartridge system to a resistivity of $>18 \mathrm{M} \Omega / \mathrm{cm}$ and purged with argon prior to the experiments. No signal was observed when the laser was fired with no gas in the helium jet.

\section{RESULTS AND DISCUSSION}

The strong transient absorption signal by $e_{\mathrm{aq}}^{-}$is used to illustrate the feasibility of using $T^{3}$ based electron generators for chemical studies. Figure 4 shows a transient decay cor-

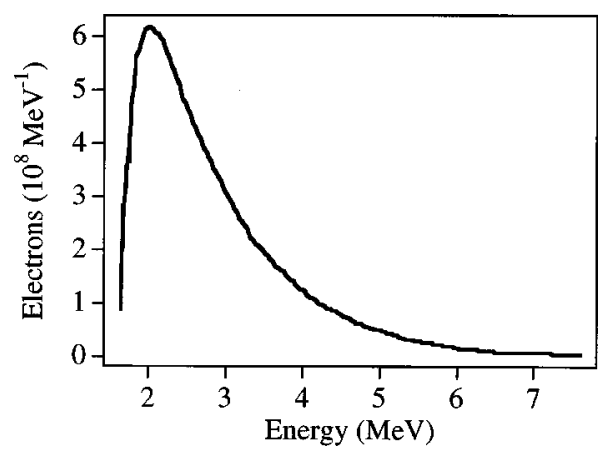

FIG. 2. Energy spectrum of the electron pulses produced with $T^{3}$ (from Ref. 4). 


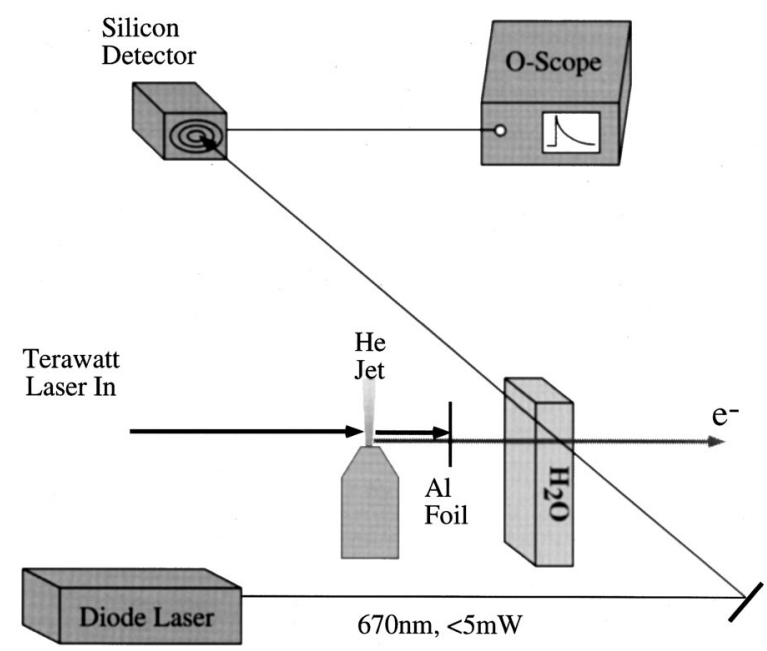

FIG. 3. Experimental setup used for measuring the microsecond decay of the $e_{\mathrm{aq}}^{-}$in water. The time resolution (determined by the detector) was 0.3 $\mu \mathrm{s}$. A $100 \mu \mathrm{m}$ thick aluminum foil was used to filter out all of the $1053 \mathrm{~nm}$ photons.

responding to an induced transmission change of $0.5 \%$ (absorbance of 0.0018 ) along with a convolution of a first-order decay with the instrument response (Gaussian FWHM 0.3 $\mu \mathrm{s})$. Transmission changes as high as $6 \%$ were observed.

All of the data obtained exhibited electrical noise that arose from two sources. The first source was from the discharge of the capacitor banks that switched the Pockel cells. The discharging capacitor banks produced a sharp rf spike that appeared at the beginning of the rising edge of the transient absorption signal (see Fig. 4). Noise from firing the supersonic jet manifested itself as a low frequency oscillation (period $\sim 7 \mu \mathrm{s}$ ). While the magnitude of both sources of noise varied from shot-to-shot, the transient behavior did not. The noise from the gas jet affected the decays at times longer than $1.75 \mu$ s after the laser pulse. For this reason, the transient data for times greater than $1.5 \mu$ s after the laser pulse were ignored. It is important to emphasize that the purpose of the current experiment is to demonstrate that $T^{3}$ based electron accelerators can produce an identifiable concentration of chemical transients, $e_{\mathrm{aq}}^{-}$in the present study.

Using Beer's Law the concentrations of the $e_{\text {aq }}^{-}$produced were calculated from the literature value for the extinction coefficient of the $e_{\mathrm{aq}}^{-}, \varepsilon_{670 \mathrm{~nm}}=1.68 \times 10^{4} \mathrm{M}^{-1} \mathrm{~cm}^{-1,8}$, the magnitude of the absorbance of $e_{\mathrm{aq}}^{-}$, and the width of the electron beam at the position of the probe laser beam (1.2 $\mathrm{mm})$. However, the true magnitude of the absorption is actually higher than what is measured in the experiment because the decay of $e_{\mathrm{aq}}^{-}$has already begun during the instrument response time. If one assumes that the decay of $e_{\mathrm{aq}}^{-}$is due to first-order kinetics, then the decay of $e_{\mathrm{aq}}^{-}$that occurs during the instrument response time can be estimated from the relation ${ }^{9}$

$$
\left[e_{\mathrm{aq}}^{-}\right]_{0}=\frac{\left[e_{\mathrm{aq}}^{-}\right]_{\tau} k_{1} \tau}{1-\exp \left(-k_{1} \tau\right)},
$$

where $\left[e_{\mathrm{aq}}^{-}\right]_{0}$ is the concentration at time $0,\left[e_{\mathrm{aq}}^{-}\right]_{\tau}$ is the concentration at time $\tau$, and $k_{1}$ is the first order rate constant. The dashed line in Fig. 4 corresponds to a convolution of the

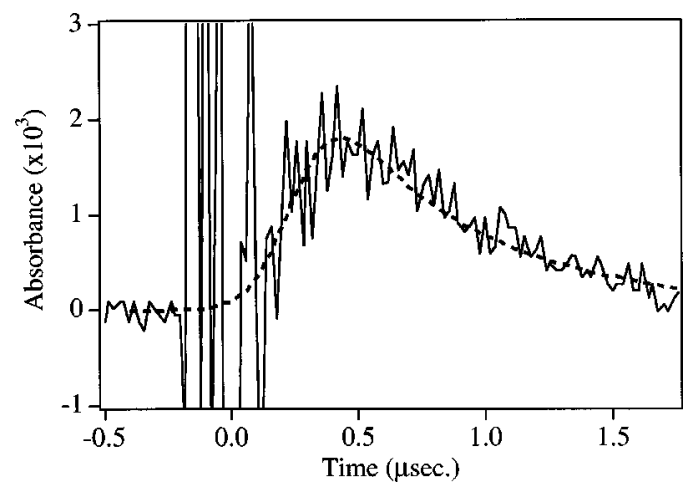

FIG. 4. Decay of the $e_{\mathrm{aq}}^{-}$generated with the electron pulses from $T^{3}$ (solid line) and a convolution of the instrument response (Gaussian FWHM 0.3 $\mu \mathrm{s})$ with a first-order decay of $1.65 \times 10^{6} \mathrm{~s}^{-1}$ (dashed line).

instrument response with a first-order decay of $k_{1}=1.65$ $\times 10^{6} \mathrm{~s}^{-1}$. This value of $k_{1}$ is consistent with the literature. ${ }^{10}$ For the decay shown in Fig. 4, Eq. (1) gives $\left[e_{\mathrm{aq}}^{-}\right]_{0}$ $\sim 1.5 \mu \mathrm{M}$. The maximum values obtained for $\left[e_{\mathrm{aq}}^{-}\right]_{0}$ in this experiment were $\sim 22 \mu \mathrm{M}$.

The charge from the laser generator can be estimated from the initial yield of the hydrated electron. Using the relationship that the yield in micromoles is approximately equal to the dose in krad times, the production rate of the hydrated electron $(2.5$ molecules/100 eV of energy deposited $^{11}$ ) and the rate at which electrons deposit energy in the medium, we can estimate the charge in the pulse. Electrons in the 1-20 MeV range deposit $2 \mathrm{MeV} / \mathrm{g}$ of material. From this, we can estimate the charge necessary to generate the data shown in Fig. 4 as $3 \times 10^{-2} \mathrm{nC}$.

It was assumed that the decay of the electron seen in Fig. 4 is first order and comes from the reaction of the electron with impurities in the system. The electron is highly reactive and reacts with oxygen at a rate of $2 \times 10^{10} \mathrm{M}^{-1} \mathrm{~s}^{-1} \cdot{ }^{12}$ This would suggest that the impurity level could be at the $10^{-4} \mathrm{M}$ level, not unreasonable for the experimental system that was used. The electron could also react with other hydrated electrons generated in the system $\left(5 \times 10^{9} \mathrm{M}^{-1} \mathrm{~s}^{-1}\right)^{12}$ or with the $\mathrm{OH}$ radical $\left(3 \times 10^{10} \mathrm{M}^{-1} \mathrm{~s}^{-1}\right) .{ }^{12}$ However, the concentrations of the $\mathrm{OH}$ and $e_{\mathrm{aq}}^{-}$generated by the electron pulse $(1.5-20 \mu \mathrm{M})$ are much smaller than would be necessary to cause the measured decay rate.

By measuring the microsecond decay of the hydrated electrons produced by the ionization of liquid water, it has been shown that the electron pulses generated with a $T^{3}$ system can provide sufficient charge to perform chemical studies. There are many advantages to using terawatt lasers for picosecond pulse radiolysis experiments. The first is that the technology already exists to generate electron pulses with a charge of a few $\mathrm{nC}^{4-6}$ Second, the cost of a terawatt laser system is much less than the cost of a photocathode-driven linac. Third and for radiation chemists the most important, the advantage is that the electron pulses are naturally synchronized with the laser pulses that generate them. This will allow the implementation of a wide variety of sensitive, laser-based detection methods that have been previously unavailable to the radiation chemists. 


\section{ACKNOWLEDGMENTS}

The work at Argonne was performed under the auspices of the Office of Science, Division of Chemical Science, U.S. Department of Energy under Contract No. W-31-109-ENG38. Experimental facilities, as well as DU and NS, were supported by the Division of Chemical Sciences, Office of Science, U.S. Department of Energy. KF and the laser facilities were supported by the National Science Foundation, Center for Ultrafast Optical Science.

${ }^{1}$ Research Needs and Opportunities in Radiation Chemistry Workshop, DOEBES, Chesterton, Indiana, 19-22 April 1988.

${ }^{2}$ Proceedings of the XIX International Linac Conference, edited by C. E. Eyberger, R. C. Pardo, and M. M. White (Argonne National Laboratory, Illinois, 1998).

${ }^{3}$ R. A. Crowell, C. D. Jonah, A. D. Trifunac, and J. Qian, in Proceedings of the XIX International Linac Conference, edited by C. E. Eyberger, R. C. Pardo, and M. M. White (Argonne National Laboratory, Illinois, 1998), p. 103; C. D. Jonah and R. A. Crowell, ibid., p. 742.
${ }^{4}$ D. Umstadter, S.-Y. Chen, A. Maksimchuk, G. Mourou, and R. Wagner, Science 273, 472 (1996).

${ }^{5}$ S.-Y. Chen, M. Krishnan, A. Maksimchuk, and D. Umstadter, Phys. Plasmas 7, 403 (2000); S.-Y. Chen, M. Krishnan, A. Maksmichuk, R. Wagner, and D. Umstadter, ibid. 6, 4739 (1999); S. P. Le Blane, M. C. Downer, R. Wagner, S.-Y. Chen, A. Maksimchuk, G. Mourou, and D. Umstadter, Phys. Rev. Lett. 77, 5381 (1996).

${ }^{6}$ G. A. Mourou, C. P. J. Barty, and M. D. Perry, Phys. Today 22 (1998).

${ }^{7}$ S. Backus, C. D. Durfee III, M. M. Murnane, and H. C. Kapteyn, Rev. Sci. Instrum. 69, 1207 (1998).

${ }^{8}$ B. D. Micheal, E. J. Hart, and K. H. Schmidt, J. Phys. Chem. 75, 2798 (1971).

${ }^{9}$ C. Capellos and A. O. Allen, J. Phys. Chem. 72, 4265 (1968).

${ }^{10}$ D. N. Nikogosyan, A. A. Oraevsky, and V. I. Rupasov, Chem. Phys. 77, 131 (1983).

${ }^{11}$ D. M. Bartels, A. R. Cook, M. Mudaliar, and C. D. Jonah, J. Phys. Chem. (in press).

${ }^{12}$ CRC Handbook of Radiation Chemistry, edited by Y. Tabata, Y. Ito, and S. Tagawa (CRC, Boca Raton, FL, 1991), p. 341. 